\title{
Interleaved Boost Power Factor Corrector Operating in Discontinuous-Inductor-Currrent Mode
}

\author{
C.H. Chan M.H. Pong \\ Department of Electrical and Electronic Engineering \\ The University of Hong Kong \\ Pokfulam Road, Hong Kong
}

\begin{abstract}
This paper presents the basic operation of the Discontinuous-Inductor-Current-Mode (DICM) boost PFC and the improvement achieved by interleaving technique. The PFC is constructed by at least two boost cells, each cell is kept running in DICM. Input current of the PFC is analyzed by numerical method and the optimum power factor is calculated in different modes of operation. The calculated result is verified by experiment and presented in graphical form with detailed discussion. The result shows that interleaving technique can alleviate the ripple current and improve the power factor. The improvement is obvious for the number of boost cells equal to 2 or 3 and the ripple alleviation is better when the PFC is operated in fixed frequency mode. The optimum power factor is improved up to 0.99 even without an input filter.
\end{abstract}

\section{INTRODUCTION}

As IEC1000-3-2 become compulsory requirement for the electronic equipment in Europe, Power Factor Corrector (PFC) is always used to reduce input harmonic currents and increase the power factor of power converters. Boost power factor corrector operated in Discontinuous-InductorCurrent Mode (DICM) is popular in low to moderate power level. The input current of this converter naturally follows the sinusoidal line voltage, therefore, the current control loop can be removed and the whole control loop is simplified. Moreover, Zero Current Switching (ZCS) is another advantage for DICM boost PFC, it reduces power losses in the power switches and the snubber circuit which suppresses reverse recovery current of the output rectifier is not required. Although DICM boost PFC has many advantages [4], it is seldom adopted in high power application. The main reason is the pulsating input current which makes the design of input filter difficult. The problem can be solved by interleaving technique [3][5] which effectively alleviates the ripple current.

A DICM boost PFC can be controlled at fixed frequency or variable frequency [4]. For the fixed frequency operation, the duty cycle (or the on-time) of the power switch is fixed within a half line cycle and there is distortion [1] in the input current waveform due to the dead time between current pulses. For the variable frequency operation, the power switch is always turned on immediately after the inductor current has reached zero and the on-time is fixed within a half line cycle.

The equations of rms value and harmonic components of the input currents are the necessary information for calculating the power factor of the boost PFC in different modes of operation. Some of these equations are already derived in [1]\&[2] and the power factor of a boost converter with fixed frequency operation is derived in [1]. However, experimental result shows that the actual power factor is much lower than the calculated value provided in [1] because it is assumed that the ripple current is completely removed by an ideal input filter. Therefore, the input current is averaged within a switching period in the calculation and the high frequency (around the switching frequency) harmonic current is ignored. To evaluate the actual performance of the DICM boost PFC (without input filter), harmonic currents over a reasonable range of frequencies should be considered. Since the input current waveform is sophisticated, special numerical method should be adopted in the analysis.

This paper presents the basic operation of the DICM boost PFC and the improvement achieved by interleaving technique. Input current of the PFC is analyzed by numerical method and the optimum power factor is calculated in different modes of operation. The calculated result is verified by experiment and presented in graphical form with detailed discussion.

\section{OPERATION OF DICM PFC AND THE INTERLEAVING TECHNIQUE}

The interleaved PFC is shown in fig.1. The PFC is constructed by at least two boost cells, all boost cells are kept running in DICM.

\section{A. Fixed Frequency Operation}

In this mode of operation, the power switches are operated in fixed switching frequency and duty cycle within a half line cycle. The inductor current in one of the boost cells is shown in fig.2, it is a series of triangular pulses with the envelop follows the input voltage.

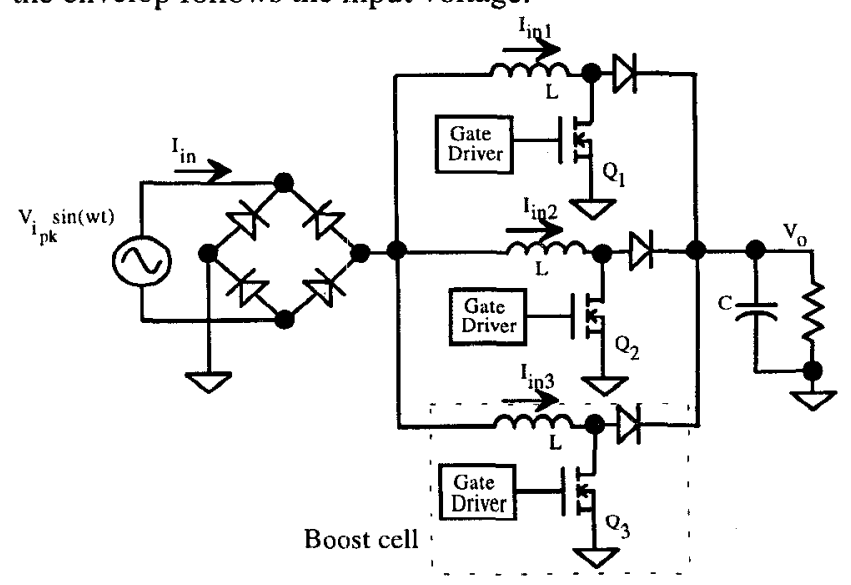

Fig. 1. Interleaved power factor corrector. 


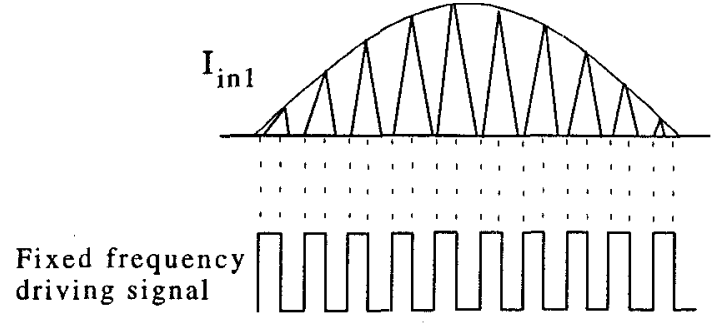

Fig. 2. Fixed frequency operation.

As the duty cycle is fixed, there is dead time in between the pulses which causes low frequency harmonic distortion [1] and the distortion is more obvious when the high frequency ripple current is filtered. To minimize the dead time, the duty cycle (D) should be set as large as possible with the condition that the converter is kept in discontinuous mode throughout the line cycle. The criterion is

$D \gtrless 1-\alpha \quad$ where $\alpha$ is the ratio of peak input voltage to output voltage $\left(\alpha=\frac{V_{i p k}}{V_{o}}\right)$

Therefore, the optimum value of duty cycle is $1-\alpha$. The solid lines in fig. 3 shows the power factor of a DICM boost cell with different values of $\alpha$ and duty cycle (the bottom x-axis). The data is obtained by applying numerical analysis to the pulsating input current, the method of analysis will be described in section III. The broken line shows the optimum power factor that can be achieved with different values of $\alpha$ (the top $x$-axis).

Take a PFC with $200 \mathrm{Vac}$ input and $400 \mathrm{~V}$ output as an example, the peak input voltage is $200 \times \sqrt{2} \simeq 280 \mathrm{~V}$ and $\alpha$ is equal to 0.7 . According to fig. 3 , the optimum power factor is 0.77 when the duty cycle is set equal to $0.3(=1-\alpha)$. If the duty cycle is set higher than 0.3 , the boost converter will go into continuous mode. In opposite, decreasing the duty cycle will significantly decrease the power factor.

\section{B. Variable Frequency Operation}

In this mode of operation, the power switches are always

\section{Irput peak voltage/Output woltage}

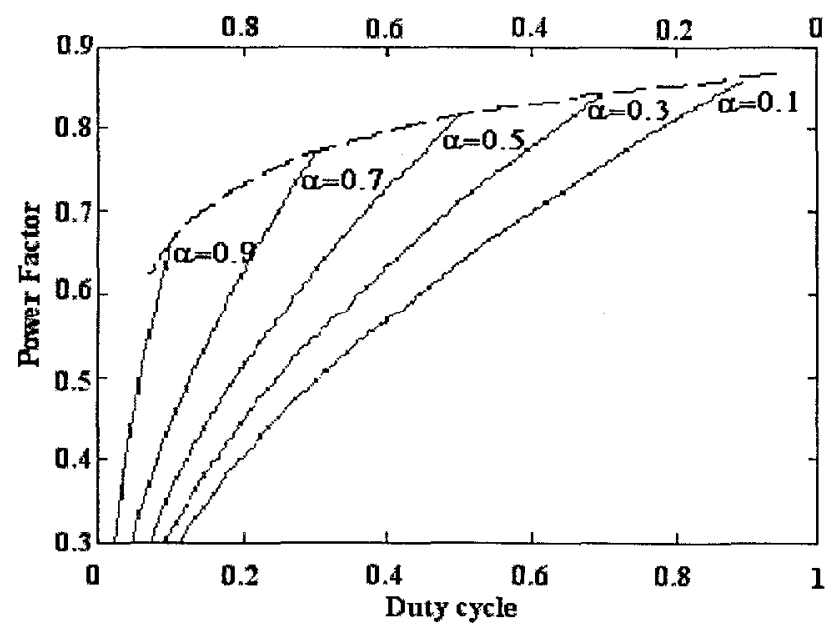

Fig. 3. Power factor of a DICM boost cell with fixed frequency operation. turned on immediately after the inductor current has reached zero. The dead time between current pulses is then eliminated and the input current is free of low frequency distortion that occurred in fixed frequency operation. Some articles designate this control method to "Borderline control" since the PFC always works in the borderline of continuous and discontinuous mode. The input current of the boost cells is shown in fig. 5 .

\section{The Interleaving Technique}

The interleaved PFC is constructed by a number of parallel connected boost cells with the same components and controlled by the same switching strategy. The driving signal of one cell is shifted in time by an appropriate amount to other cells as shown in fig.4. As the input current of the whole PFC is the sum of the inductor current of all boost cells, the resultant ripple current is reduced.

For the variable frequency operation, there are two methods to apply the interleaving technique. Method 1 uses individual controllers to control the boost cells separately and the boost cells are kept running in borderline mode by their own controllers. To effectively cancel the ripple current, the driving signals from the controllers are synchronized by setting the driving signals to zero at the zero cross of the input voltage and then the signals are restarted with different delay as shown in fig. 5 .

However, this method is difficult to implement because the controllers do not have exactly same characteristic, that

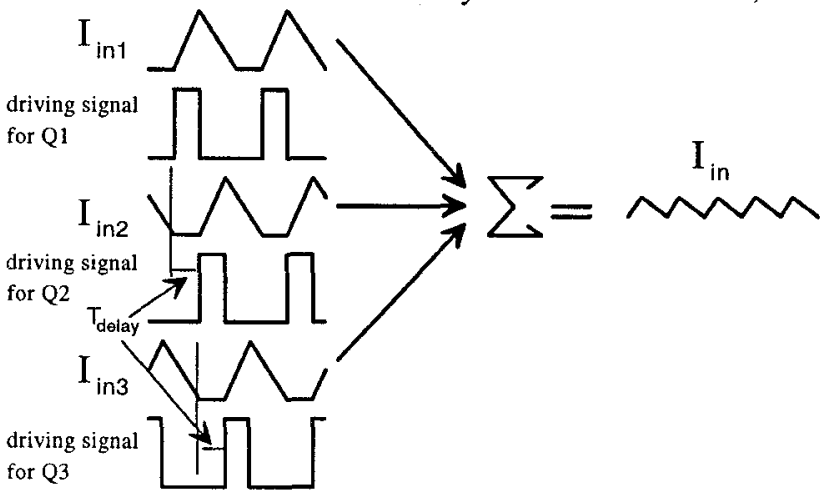

Fig. 4. Interleaving technique

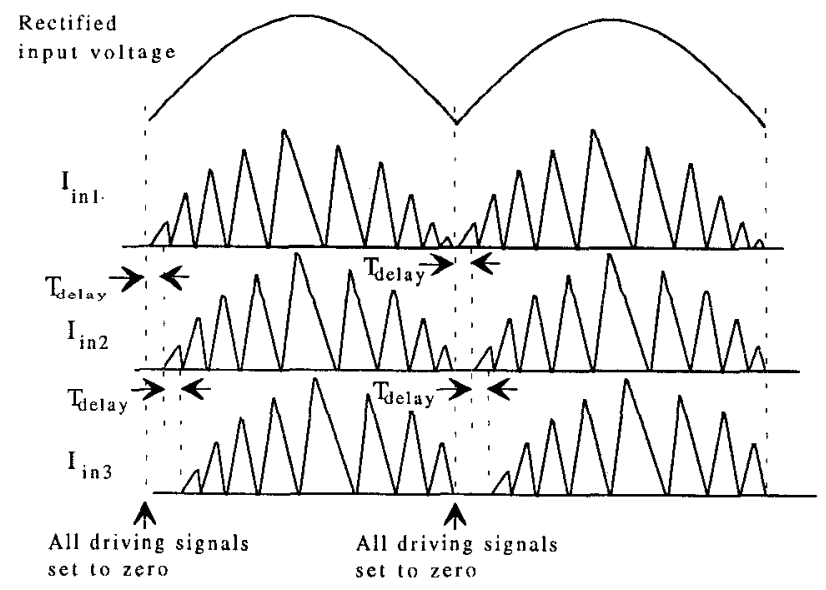

Fig. 5. Interleaving technique for variable frequency operation. 
means different drift of current pulses can happen in different cells. The drift of the current waveform will greatly affect the ripple cancellation and deviate the result.

Method 2 is a more practical method which use only one controller to control the first PFC cell. The other PFC cells are controlled by the same driving signal from the controller with time delay. Although the PFC cells are not exactly kept in borderline mode except the first cell, the derivation is so little that all PFC cells are apparently running in borderline mode.

\section{THE METHOD OF ANALYSIS}

The input current waveform is analyzed by dividing it into triangular pulses as shown in fig. 6. By summation of the triangular pulses, the input current waveform can be reconstructed. To simplify the analysis, the following assumptions are made: 1) The input voltage is constant over one switching period. 2) The switching frequency is much higher than the line frequency. 3) The output ripple voltage is negligible and the output voltage is constant. 4) The on-time of the power switch $\left(\mathrm{T}_{\mathrm{on}}\right)$ is constant within a half line cycle. 5) The inductance of the inductors $L$ in all boost cells are equal.

For the fixed frequency operation, the total number of pulses within a line cycle is equal to $K=\frac{T}{T_{s}}$. To make the equations of the input current clearer, we let $T_{1}(k)$ be the starting time of the k-th pulse, $\mathrm{T}_{2}(\mathrm{k})$ be the time for the $\mathrm{k}$-th pulse to reach its peak value and $T_{3}(k)$ be the time for the $\mathrm{k}$-th pulse to fall to zero.

$T_{1}(k)=k T_{s}, \quad T_{2}(k)=k T_{s}+T_{o n}$, and

$T_{3}(k)=k T_{s}+T_{o n}+T_{d}(k)$ for $\mathrm{k}=0,1,2 \ldots ., \mathrm{K}-1$

where $T_{d}(k)$ is the conduction time of the output rectifier.

The equation of the triangular pulse is

$i_{k}(t)=\frac{V_{i_{p k}} \sin w T_{1}(k)}{L}\left(t-T_{1}(k)\right)$ for $T_{1}(k)<t \leqslant T_{2}(k)$

$i_{k}(t)=\frac{V_{i_{p k}} \sin w T_{1}(k)}{L} T_{o n}+\frac{\left|V_{i_{p k}} \sin w T_{1}(k)\right|-V_{o}}{L}\left(t-T_{2}(k)\right)$

$$
\text { for } T_{2}(k)<t \leqslant T_{3}(k)
$$

$i_{k}(t)=0$ for $T_{3}(k)<t \leqslant T_{1}(k+1)(2 \mathrm{c})$

The input current is equal to the sum of the triangular pulses.

i.e. $i_{\text {in }}(t)=\sum_{k=0}^{K-1} i_{k}(t)$

for fixed frequency operation.

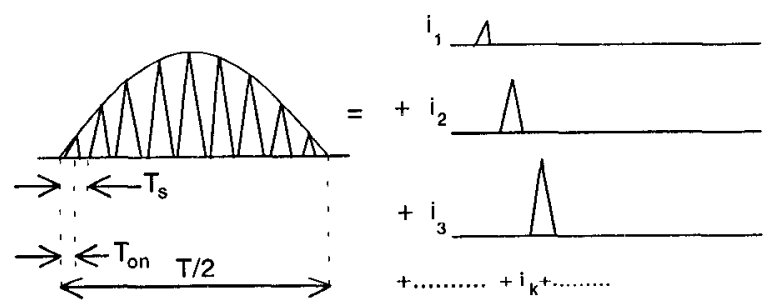

Fig. 6. The method of analysis
For variable frequency operation, the dead time between the triangular pulses is eliminated. The equations of the triangular pulses are basically same as that of fixed frequency operation, however the variables $T_{1}(k)$ to $T_{3}(k)$ should be redefined and $(2 c)$ is rejected.

$T_{1}(k)=T_{1}(k-1)+T_{o n}+T_{d}(k-1), T_{2}(k)=T_{1}(k)+T_{o n}$, and

$T_{3}(k)=T_{2}(k)+T_{d}(k)=T_{1}(k+1) \quad$ for $k=0,1,2 \ldots$.

Although the total number of pulses within a line cycle is unknown in this case, we can use the condition $(t \geq T)$ to indicate the end of summation in the equation of input current. Therefore,

$i_{\text {in }}(t)=\sum_{t=0}^{t \geq T} i_{k}(t)$

for variable frequency operation.

Applying the interleaving technique, the triangular pulses for the second boost cell start with a delay $T_{\text {delay }}$ and the triangular pulses for the third boost cell start with a delay 2 $\times \mathrm{T}_{\text {delay }}$ and so on, then the currents flow into the inductors of the boost cells are defined as

$i_{\text {in } 1}(t)=\sum_{k=0}^{K-1} i_{k}(t)$

$i_{\text {in } 2}(t)=\sum_{k=0}^{K-1} i_{k}\left(t-T_{\text {delay }}\right), \quad$ and

$i_{i n 3}(t)=\sum_{k=0}^{K-1} i_{k}\left(t-2 \times T_{\text {delay }}\right)$

And the resultant input current will be

$i_{\text {in }}(t)=i_{\text {in } 1}(t)+i_{\text {in } 2}(t)+i_{\text {in } 3}(t)+\ldots .$.

Using (7), we analyzed the input current by Fourier analysis and calculated the power factor of the PFC.

\section{ANALYSIS AND RESULT}

\section{A. Fixed Frequency Operation}

It is intuitively known that the most effective way to minimize the ripple current is setting $T_{\text {delay }}=\frac{T_{s}}{N}$ (where $\mathrm{N}$ $=$ no. of boost cells) because the current pulses of all boost cells can be evenly distributed within a switching period.

With the above information, the input current waveform of the PFC with different number of cells is analyzed by Fourier analysis under the following conditions: 1) The duty cycle is set equal to $1-\alpha$, then the power factor is optimum for the corresponding value of $\alpha$. 2) $T_{\text {delay }}$ is set equal to $\frac{T_{s}}{N}$ with the aforementioned reason.

The result is presented in fig. 7. and the lowest curve is same as the broken line in fig. 3 which shows the power factor of the non-interleaved PFC. The result shows that: 1) For $\mathrm{N}=1$, power factor is low and continuously decrease as $\alpha$ increase. 2) For $\mathrm{N}=2$ or above, power factor is much improved and the improvement is obvious when $\mathrm{N}$ change from 1 to 2 but it is not obvious when $N$ is further increased. 3) The power factor of the interleaved PFC is not continuously decreased as $\alpha$ increase, this is different from the case of the non-interleaved one. The optimum points for different values of $\mathrm{N}$ are shown in Table I. It shows that an 


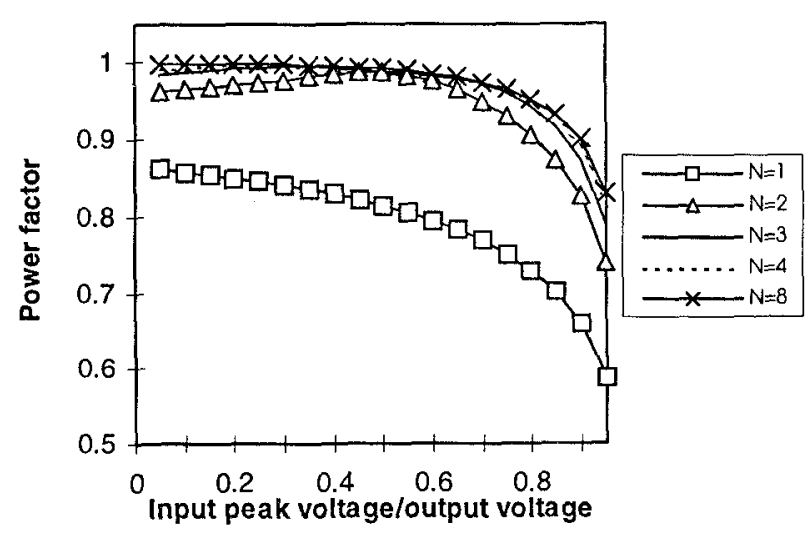

Fig. 7. Optimum power factor of the interleaved PFC

TABLE I

MAXIMUM VALUE OF POWER FACTOR OF INTERLEAVED PFC (FIXED FREQUENCY OPERATION)

\begin{tabular}{ccc}
\hline $\mathrm{N}$ & Value of $\alpha$ when PF is maximum & Maximum PF \\
\hline 1 & near zero & 0.864 \\
2 & 0.5 & 0.987 \\
3 & 0.33 & 0.997 \\
4 & 0.25 & 0.998 \\
8 & 0.125 & 0.999 \\
\hline
\end{tabular}

interleaved PFC with $\mathrm{N}$ boost cells can give a maximum power factor when $\alpha$ equal to $1 / \mathrm{N}$.

To verify the above result, three boost cells was built and tested under different input voltages. The test result is compared with the calculated result in fig. 8 which shows that the calculation agrees with the experimental result. The input currents of the PFC with different number of cells are shown in fig. 9 for comparison. Obviously, the ripple current is much reduced using the interleaving technique.

To evaluate the actual contribution of interleaving technique in ripple alleviation, the analysis should focus on the high frequency ripple alone. Therefore, the dead time distortion is extracted and eliminated in the analysis by the following procedure.

The actual input current $I_{i n}$ is composed by the high frequency and low frequency harmonic currents, the former is due to the ripple current and the latter is due to the dead time distortion. The distortion factor of $\mathrm{I}_{\text {in }}$ is defined as

$\mu_{\text {in }}=\frac{I_{1}}{I_{\text {in, rms }}}=\frac{I_{1}}{\sqrt{I_{1}^{2}+I_{\text {low }}+I_{\text {high }}}}$

where $I_{1}=$ the rms value of fundamental current

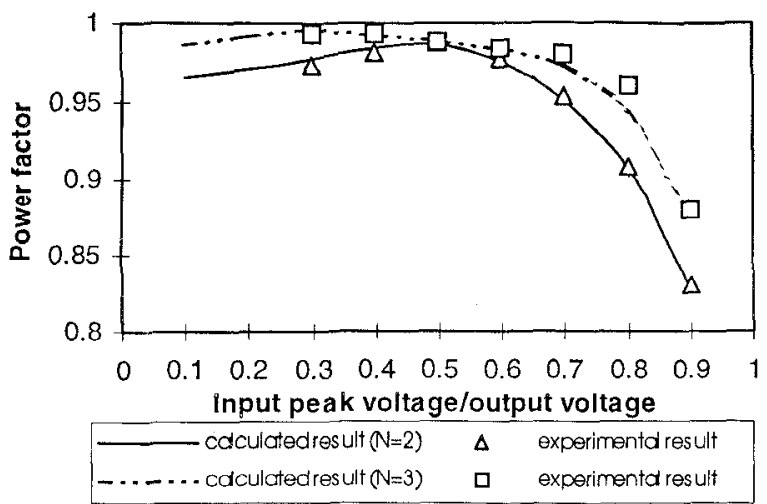

Fig. 8. Comparison of calculated result and experimental result.
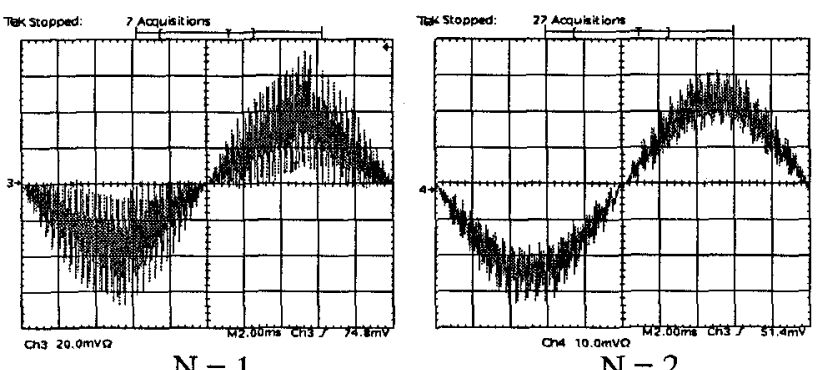

$\mathrm{N}=1$

$\mathrm{N}=2$

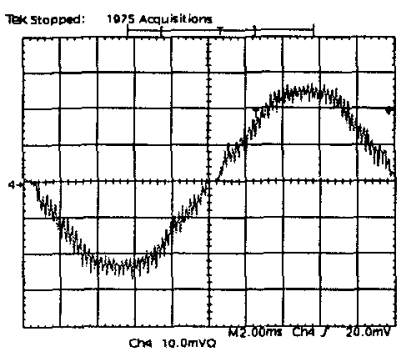

$\mathrm{N}=3$

Fig. 9. Input current of interleaved boost PFC.

$I_{i n, \text { rns }}=$ the rms value of the input current

$\mathrm{I}_{\text {low }}=$ the sum of square of the harmonics due to dead time distortion $=I_{2}^{2}+I_{3}^{2}+I_{4}^{2}+\ldots \ldots$.

$I_{\text {high }}=$ the sum of square of the harmonics due to the high frequency ripple

Note that

Power Factor $=$ Distortion Factor $\times$ Displacement Factor and Displacement Factor is nearly equal to one, therefore, the Power Factor is equal to the Distortion Factor in this case. (That means fig. 7 can also used to present the value of $\mu_{\text {in }}$ )

The input current and power factor of a single cell PFC are defined in [1] as follow.

$I_{\text {in }}(t)=\frac{V_{i_{p k}} D^{2} T_{s}}{2 L} \frac{\sin w t}{1-a \sin w t}$ and

$P F=\frac{\sqrt{2}}{\sqrt{\pi}} \cdot \frac{y}{\sqrt{z}}$

where $y=\int_{0}^{\pi} \frac{\sin ^{2} \theta}{1-a \sin \theta} d \theta$ and $z=\int_{0}^{\pi}\left[\frac{\sin \theta}{1-a \sin \theta}\right]^{2} d \theta$

Since the high frequency ripple is ignored in (9) and (10), the current contains only the low frequency harmonics due to the dead time distortion. The distortion factor for the low frequency harmonics is defined as

$\mu_{l o w}=\frac{I_{1}}{\sqrt{I_{1}^{2}+I_{l o w}}}$

As power factor is equal to distortion factor, $\mu_{\text {low }}$ can be directly calculated by (10) because the equation refers to the low frequency harmonics only.

Combine (8) and (11),

$\frac{\mu_{\text {low }}}{\mu_{\text {in }}}=\frac{\sqrt{I_{1}^{2}+I_{\text {low }}+I_{\text {high }}}}{\sqrt{I_{1}^{2}+I_{\text {low }}}}$

$\frac{\sqrt{I_{\text {high }}}}{I_{1}}=\sqrt{\frac{1}{\mu_{\text {in }}^{2}}-\frac{1}{\mu_{\text {low }}^{2}}}$ 
The left hand side of (12) is the ratio of high frequency harmonics to the fundamental current (ripple ratio) and this ratio is suitable for indicating the percentage of ripple current in the input current.

Ripple ratio $(\mathrm{R})=\frac{\sqrt{I_{\text {high }}}}{I_{1}}=\sqrt{\frac{1}{\mu_{i \text { in }}^{2}}-\frac{1}{\mu_{\text {low }}^{2}}}$

where $\mu_{\text {in }}$ is obtained in the Fourier analysis of the input current and $\mu_{\text {low }}$ can be calculated by (10).

To clearly observe the improvement due to interleaving, the ripple ratios of the PFC with different number of cells are compared with the non-interleaved PFC (i.e. $\mathrm{N}=1$ ). The ripple attenuation (RA) is then defined as

$R A_{N}=\frac{R_{N}}{R_{1}}$

Fig. 10 shows the ripple attenuation of the interleaved PFC with different number of cells and different values of $\alpha$ and the optimum ripple attenuation are shown in Table II.

\section{B. Variable Frequency Operation}

For the variable frequency operation, similar numerical analysis has been carried out. Different values of $T_{\text {on }}$ are tried in the analysis of non-interleaved PFC. The result shows that the power factor is equal to 0.866 as $T_{\text {on }}$ trends to zero and it is nearly constant over a wide range of $T_{\text {on }}$. However, the power factor is deviated from 0.866 if $T_{o n}$ is longer than $\frac{\text { line period }}{25}$. It is because the first two assumptions (the input voltage is constant over one switching period and the switching frequency is much higher than the line frequency) are not held as $\mathrm{T}_{\text {on }}$ has a comparative length to the line period. On the other hand, the value of $\alpha$ has no significant effect on the power factor and the distortion is only due to the high frequency ripple current because the dead time distortion is eliminated in this case.

When the interleaving technique is applied to the PFC using method 1, it is found that the power factor is depended on the delay between the driving signals but not $\alpha$. The result is shown in fig. 11. Obviously, the power factor is maximum for $T_{\text {delay }}=\frac{T_{o n}}{N}$. The optimum points for different values of $\mathrm{N}$ are shown in Table III.

Same as the case of fixed frequency operation, the power factor is equal to the distortion factor. In this case, the distortion factor of $I_{\text {in }}$ is defined as

$\mu_{\text {in }}=\frac{I_{1}}{I_{\text {in, ms }}}=\frac{I_{1}}{\sqrt{I_{1}^{2}+I_{\text {high }}}}$

and the ripple ratio is then equal to

$\mathrm{R}=\frac{\sqrt{I_{\text {high }}}}{I_{1}}=\sqrt{\frac{1}{\mu_{\text {in }}^{2}}-1}$

Again, the ripple attenuation is calculated by (14) and the optimum values are shown in Table IV.

TABLE II

OPTIMUM RIPPLE ATTENUATION
(FIXED FREQUENCY OPERATION)

\begin{tabular}{cc} 
& (FIXED FREQUENCY OPERATION) \\
\hline $\mathrm{N}$ & Optimum Ripple Attenuation \\
\hline 2 & $14.7 \%$ \\
3 & $8.5 \%$ \\
4 & $4 \%$ \\
8 & $1 \%$ \\
\hline
\end{tabular}

TABLE III

Maximum Value of Pover Factor of INTERLEaved PFC (VARIABLE FrEQUENCY OPERATION - METHOD 1)

\begin{tabular}{ccc}
\hline $\mathrm{N}$ & Value of $\mathrm{T}_{\text {delay }} / \mathrm{T}_{\text {on }}$ whien PF is maximum & Maximum PF \\
\hline 1 & - & 0.866 \\
2 & 0.5 & 0.986 \\
3 & 0.33 & 0.998 \\
4 & 0.25 & 0.999 \\
8 & 0.125 & 0.999 \\
\hline
\end{tabular}

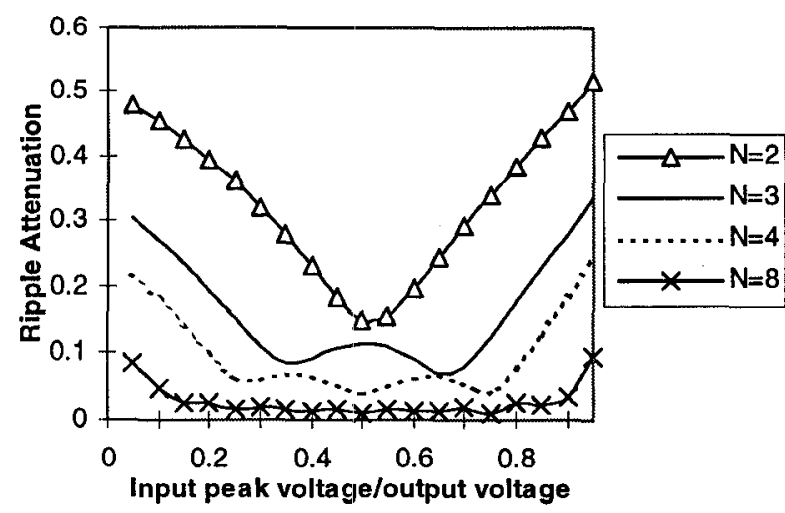

Fig. 10. Ripple Attenuation (fixed frequency operation)

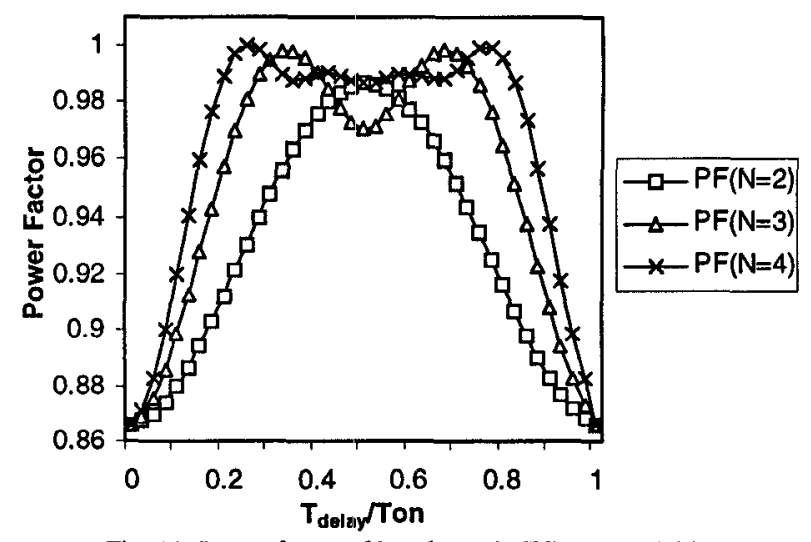

Fig. 11. Power factor of interleaved PFC with variable frequency operation - Method 1.

A completely different result is obtained using method 2 . It is found that the power factor is depended on both $\alpha$ and the ratio $\frac{T_{\text {delay }}}{T_{\text {on }}}$. Fig. 12 and fig. 13 show the power factor of the interleaved $\mathrm{PFC}$ with $\mathrm{N}=2$ and $\mathrm{N}=3$ respectively. The optimum values of ripple attenuation are shown in Table $\mathrm{V}$.

\section{DISCUSSION}

Using the above result, engineers can optimize the number of boost cells in designing PFC for different application. Although the interleaving technique increases the component count, the actual increase of cost may be not significant. It is because using more boost cells can share the current flow in the inductors and power switches so the lower current rating devices (lower price) can be adopted. Actually, some high power PFC use more than one power switches and output rectifiers because the high current rating device is not available in the market. 
TABLE IV

OPTIMUM RIPPLE ATTENUATION

(VARIABLE FREQUENCY OPERATION - METHOD 1)

\begin{tabular}{cc}
\hline $\mathrm{N}$ & Ripple Attenuation \\
\hline 2 & $29.2 \%$ \\
3 & $11 \%$ \\
4 & $7.8 \%$ \\
8 & $7.8 \%$ \\
\hline & TABLE V
\end{tabular}

OPTIMUM RIPPLE ATTENUATION

(VARIABLE FREQUENCY OPERATION - METHOD 2)

\begin{tabular}{ccccc}
\hline $\mathrm{N}$ & $\alpha$ & $\begin{array}{c}\text { Value of } \mathrm{T}_{\text {delay }} / \mathrm{T}_{\text {on }} \\
\text { when PF is maximum }\end{array}$ & $\begin{array}{c}\text { Maximum } \\
\text { PF }\end{array}$ & $\begin{array}{c}\text { Ripple } \\
\text { Attenuation }\end{array}$ \\
\hline 2 & 0.1 & 0.5 & 0.966 & $46.4 \%$ \\
& 0.3 & 0.7 & 0.981 & $34.2 \%$ \\
& 0.5 & 0.9 & 0.99 & $24.7 \%$ \\
& 0.7 & 1 & 0.968 & $44.9 \%$ \\
& 0.9 & 1 & 0.923 & $72.2 \%$ \\
\hline 3 & 0.1 & 0.4 & 0.988 & $27.1 \%$ \\
& 0.3 & 0.5 & 0.997 & $13.4 \%$ \\
& 0.5 & 0.6 & 0.994 & $19.1 \%$ \\
& 0.7 & 0.9 & 0.993 & $20.6 \%$ \\
& 0.9 & 1 & 0.952 & $55.7 \%$ \\
\hline
\end{tabular}

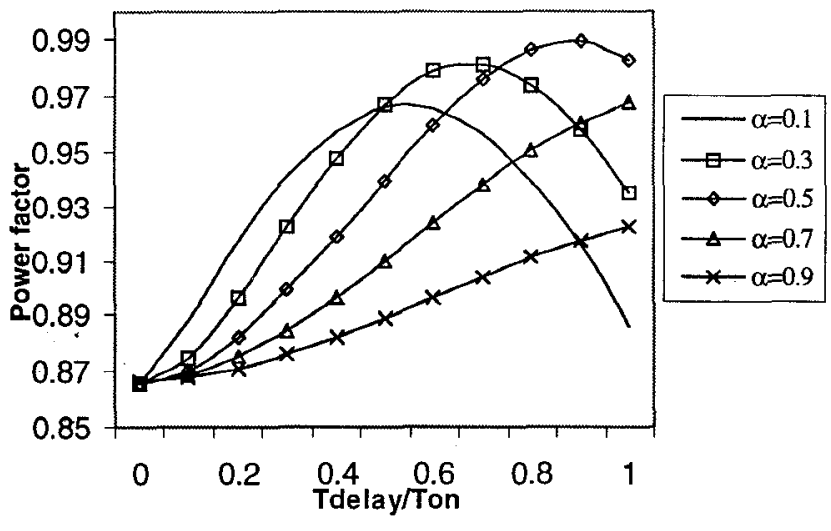

Fig. 12. Power factor of interleaved PFC $(\mathrm{N}=2)$ with variable frequency operation - Method 2.

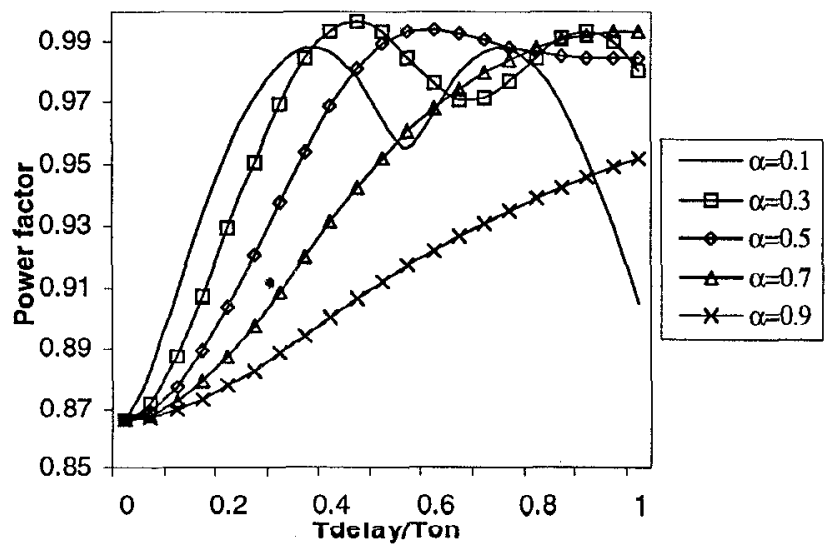

Fig. 13. Power factor of interleaved PFC $(\mathrm{N}=3)$ with variable frequency operation - Method 2

However, the effect of interleaving technique is obvious only for $\mathrm{N}$ equal to 2 or 3 . Thus it is a pitfall for one who think "more cells get better result". In fact, the high frequency ripple current can be effectively reduced using 2 or 3 boost cells.
TABLE VI

COMPARISON OF RIPPLE ATTENUATION

\begin{tabular}{cccc}
\hline & $\begin{array}{c}\text { Fixed frequency } \\
\text { operation }\end{array}$ & \multicolumn{2}{c}{$\begin{array}{c}\text { Variable frequency } \\
\text { operation }\end{array}$} \\
\hline & & Method 1 & Method 2 \\
\hline Range of Ripple & $14.7 \%-50 \%$ & $29.2 \%$ & $\begin{array}{c}24.7 \%- \\
72 \%\end{array}$ \\
Attenuation (N=2) & & & $11 \%$ \\
\hline Range of Ripple & $8.5 \%-30 \%$ & $13.4 \%-$ \\
Attenuation (N=3) & & & $55.7 \%$ \\
\hline
\end{tabular}

Comparing the result (table VI), the interleaved PFC with fixed frequency operation gives the best ripple attenuation but the attenuation is varied with the value of $\alpha$, Also, the power factor is lowered by the dead time distortion which is a shortcoming of fixed frequency operation.

For variable frequency operation, the ripple attenuation is constant for the interleaved PFC using method 1. That means the PFC can keep the power factor stable in different input line voltage and output loading. However, the controller is very difficult to implement because of the reason stated in section $\mathrm{II}(\mathrm{C})$. Method 2 is a more practical scheme to control the interleaved PFC with variable frequency operation, but the advantage of method 1 is lost. In general, the controller for variable frequency operation is more complicated because it requires sensing the current through the inductor (or output rectifier). Therefore, fixed frequency operation is recommended unless the dead time distortion is intolerable.

\section{CONCLUSION}

An accurate input current waveform analysis is carried out to investigate the power factor of the interleaved DICM PFC. The waveform is analyzed by numerical method in both fixed frequency and variable frequency operation. The optimum power factor is calculated and the result shows that interleaving technique can alleviate the ripple current and improve the power factor. The improvement is obvious for the number of boost cells equal to 2 or 3 and the power factor can be improved up to 0.99 even without an input filter. Considering the ripple attenuation and complexity of the controllers, fixed frequency operation is recommended to adopted in the interleaved PFC.

\section{ACKNOWLEDGMENT}

Special thanks to ASTEC Custom Power (H.K.) for the help and advice on the research.

\section{REFERENCES}

[1] Kwang-Hwa Liu and Yung-Lin Lin, "Current waveform distortion in power factor correction circuits employing discontinuous-mode boost converters", Proceedings of PESC '89, pp. 825-829.

[2] Richard Redl and Laszlo Balogh, " Rms, dc, peak, and harmonic currents in high-frequency power-factor correctors with capacitive energy storage", Proceedings of APEC '92, pp. 533-540.

[3] Laszlo Balogh and Richard Redl, "Power-factor correction with interleaved boost converters in continuous-inductor-current mode", Proceedings of APEC '93, pp. 168-174.

[4] Bill Andreycak, "Controlled On-time, Zero Current Switched Power Factor Correction Technique" Proceedings of HFPC June 1991, pp. 46-60.

[5] Brett A. Miwa, David M. Otten and Martin F. Schlecht, "High efficiency power factor correction using interleaving techniques", Proceedings of APEC ' 92 , pp. 557-568. 